\title{
Dynamic Management Architecture for Project Based Production
}

\author{
Akira Tsumaya ${ }^{1}$, Yuta Matoba ${ }^{2}$, Hidefumi Wakamatsu ${ }^{2}$ and Eiji Arai ${ }^{2}$ \\ 1 Kobe University, Department of Mechanical Engineering, Graduate \\ School of Engineering \\ Rokkodai 1-1, Nada, Kobe 657-8501, Japan \\ tsumaya@mech.kobe-u.ac.jp \\ WWW home page: http:/www.mech.kobe-u.ac.jp/ tsumaya/index-e.html \\ 2 Osaka University, Division of Materials and Manufacturing Science, \\ Graduate School of Engineering \\ Yamadaoka 2-1, Suita, Osaka 565-0871, Japan \\ \{matoba,wakamatu, arai\}@mapse.eng.osaka-u.ac.jp, \\ WWW home page: http://www6.mapse.eng.osaka-u.ac.jp/index-eng.html
}

\begin{abstract}
The production system where production facilities and products are widely distributed like a construction production project causes problems by combining and communicating production facilities mutually. There is a limit of the traditional approaches, where the system obtains the product information from the production facilities. In this paper, the production system architecture which manages the dynamic scheduling and material handling by using parts and packets unification technology is proposed. First, the architecture of the production based production with parts and packets unification technology is proposed. In order to treat both scheduling and material handling, not only the information of "what part" and "when exists" but also "where exists" is used. Then, a pilot system is developed and applied to the case studies about the production scheduling and material handling system in a factory. The results of the case studies show the feasibility of proposed production system using parts and packets unification approach.
\end{abstract}

\author{
Keywords \\ Dynamic Management, Parts and Packets Unification, Project Based \\ Production, Scheduling, Material Handling
}




\section{Introduction}

A complicated production system is needed to satisfy various requirements to production these days. While it is more and more difficult to manage such various requirements by centralized production management system, the concept of an autonomous distributed production system is proposed as what can adopt to such circumstances. Therefore, numerous researchers have proposed concept of autonomous \& distributed production systems in recent years. Many of them focused on product facilities, and constructed intelligent system using network structure, and are available when production facilities and products exist in the limited space like automated factories [1-6].

On the other hand, the production system where production facilities and products are widely distributed like a construction production project causes problems by combining and communicating production facilities mutually. For example, it is difficult to grasp the situation of the production in real time, or the processing performed during the transfer between facilities. In the case of the cooperative work by several different companies, it is more difficult to combine facilities in a network. There is a limit of the traditional approaches, where the system obtains the product information directly but obtains it from the production facilities.

In recent years, RFID tags which can store various data to parts are utilized, and can be used from a viewpoint of cost, size, and memory size. Many application of RFID are introduced and some of them are popular these days [7, 8]. Therefore, the real-time information about various production activities are directly obtained/read/written by using RFID tags which stuck on produced objects.

In this paper, the production system architecture which manages the dynamic production by using parts and packets unification technology is proposed.

\section{Concept of Parts and Packets Unification System}

For constructing dynamic and flexible production system, it is important to comprehend the state of the product in the production process. To comprehend production state, we need some kind of information. Those are;

- What the product is

- When the product is started or finished in production processes

- Where the product is

- What kind of state the product have

Producing part and packet unification architecture, which is realized by RFID tag attached in the producing part, can store and handle such kind of information $[9,10]$.

The concept of a dynamic production scheduling system using parts and packets unification technology is shown in Fig. 1. The RFID tag is attached in the producing object, and ID number and the present status are written in it. Status is rewritten according to becoming a unit from materials. The RFID tag transmits ID number, current position, and status through network system whenever it passes through the gate provided in the inside of the factory, the construction site, in the transfer route between them, and the entrance of a warehouse, etc. 


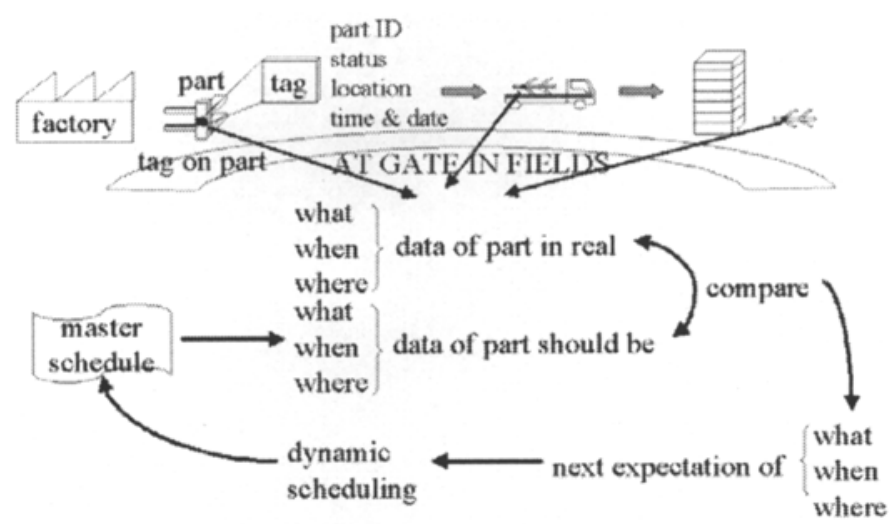

Fig. 1. The concept of a dynamic production scheduling system using parts and packets unified architecture.

As for the production planning system, progress management of the production project is performed by the scheduling system after a master schedule is drawn up. The scheduling system can show the position and status in which each producing object should exist. By comparing with real-time information on each producing object transmitted through network system to the master schedule, a gap between the master schedule and an actual state is detectable. According to the quantity of a gap, re-scheduling is performed when the system judges it is needed.

Using this architecture, a production system can recognize the actual status of all producing parts in real time. The following effects are expected to an autonomous distributed production system.

- Assistance of intelligent facilities (AGV, machine tool, robot, etc.)

- Increasing / free setting of information $1 /$ O place

- Improvement of information accuracy in real time

- Improvement of trigger accuracy on dynamic scheduling

- Expansion of trigger range on dynamic scheduling

\section{System architecture}

The architecture of dynamic production management system using parts and packets unification technology is explained on this section. Figure 2 shows the system architecture in a factory. The network system built by the conventional intelligent manufacturing system has combined production facilities, such as production cells and stations. Meanwhile, in the proposed system, the information that each part have in RFID tag can be exchanged with network system through the gate provided in each production facilities. For example, at the gate provided in the outlet of a processing facility, the status of parts is rewritten according to the production processing. 
If the memory capacity of RFID tag will become large in the near future, it is also possible to compose a network system only with RFID tags attached to the parts by storing processing processes and work information in RFID tag, and exchanging information about them with production facilities. However, since the present RFID tag has not satisfactory memory capacity, the proposed system hybridized the network system which combines intelligent production facilities, and parts and packets unification technology. Process and work information are transmitted to production facilities from the scheduling and CAM systems through the network. The part ID in the RFID tag is checked and after processing process, the status of the part is rewritten and is also sent to the CAM system.

Status information in RFID tag changes by the production process such as Fig. 3 . Condition of the production process is grasped by the dynamic production management system based on information of parts such as process/delivery start/end time, position, etc. For example, processing time is calculated with process end time and process start time. Cell ability is recognized from this calculated data.

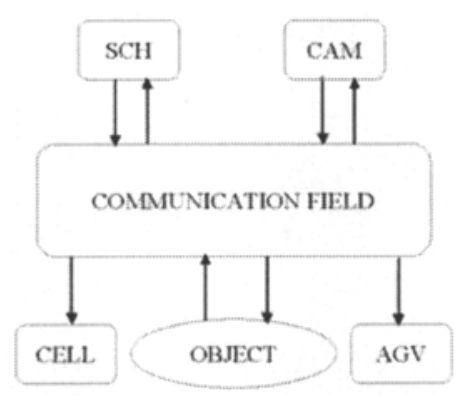

Fig. 2. The concept of a dynamic production system architecture in a factory

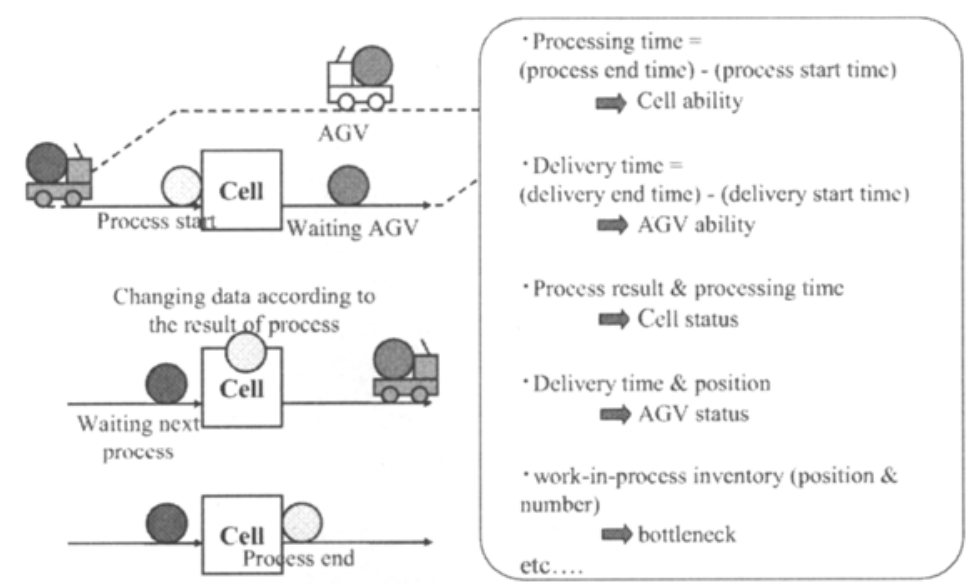

Fig. 3. Parts and packets unified data in a factory 


\section{Application to Dynamic Scheduling}

In this section, an application system of production process within a factory is explained as an example of the dynamic production system. The components of the system are shown in Fig. 4. This example has the following preconditions:

- This factory has following facilities; those are five machining cells, two AGVs (Automated Guided Vehicle), and storage. Five machining cells and storage are arranged in circulate. It is assumed that the conveyance capabilities of two AGV are equal, and it moves in the orbit with one way.

- Previously, the initial value of machining ability on each machining cell is set up. If the ability of machining cell changes during the production, proposed scheduling system calculates new value using the started and finished processing time data obtained from producing objects.

- The order of processing jobs is determined beforehand, and cannot be changed. Part processing which consists of several jobs is prepared. The work data is also provided. According to this work data, each job is given with processing order. Moreover, the processible cells for each jobs are beforehand decided according to the processing type.

- Each producing object has processing geometrical form and volume as data, and machining time is calculated based on these information.

- Scheduling is performed according to the SPT (Shortest Processing Time First) rule which makes the shortest average processing time in the job shop.

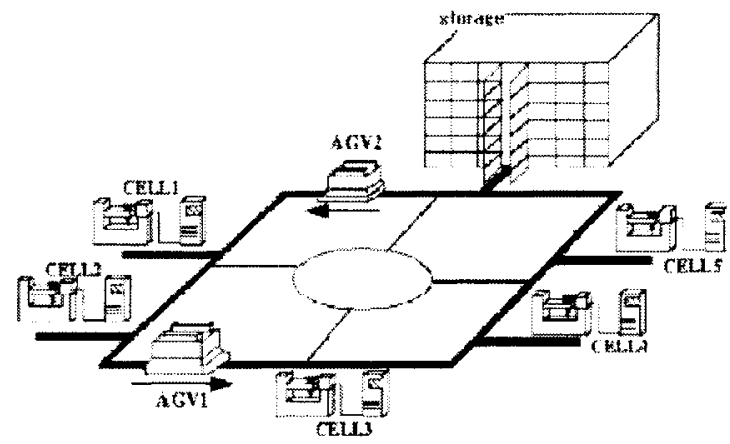

Fig. 4. The components of an application system

Some virtual simulation was performed using the system. Here, the making rescheduling corresponding to the ability fall of a machining cell is introduced as an example case. This system checks number of waiting parts in front of each machining cell derived from position data of the part, and performs re-scheduling when the number of waiting part is rather than a setting number. On re-scheduling process, the ability of the cell is re-calculated by using information on the processing start time and finish time from latest processed part. The Gantt chart of the simulation is shown in Fig. 5. As these results, the system worked effectively against changing state of production facility. 

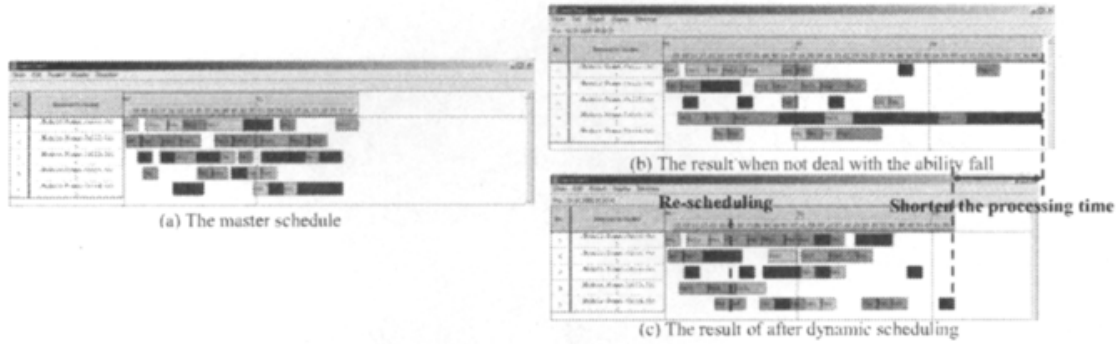

Fig. 5. The Gantt chart of the simulation

\section{Application to Material Handling and Scheduling}

The system architecture is applied to the management of both material handling and assembly processes. Here, a construction site is taken up as an example case study of the material handling and scheduling for assembly processes.

Figure 6 (a) shows the virtual construct site model of simplified simulated wall construction process on the floor. This model has the following preconditions:

- The wall of the floor is constructed by three companies; A, B, C. Each company makes the wall by using different component materials. There are three component depositories on the floor and each company occupies one of them.

- A horizontal inner wall is constructed by company A. A vertical inner wall is made by company $\mathrm{B}$. A wall surrounding the floor is constructed by company $\mathrm{C}$.

- Each company can construct the wall only when the route between the place of the component depository and the place of the wall are constructed is secured.

Figure 6 (b) shows the snap shot of which warning has gone out when the system detects that it becomes impossible the work of company B because of no access route to the constructing place from the material depository. Like this example, from present position information of component materials and state of the construction progress, it is possible to control the material handling and assembly schedule by checking process before the problem occurred.

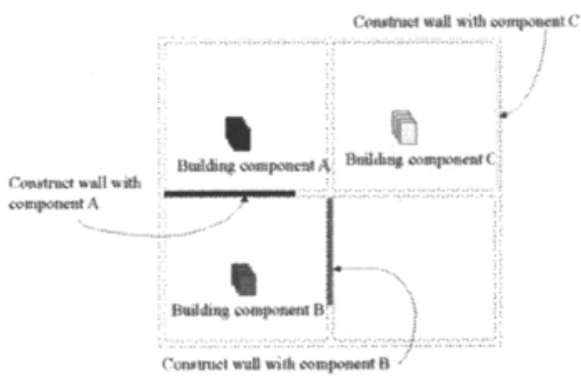

(a) Simulation model

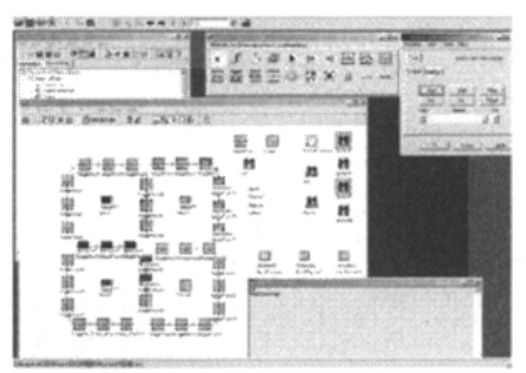

(b) result of the simulation

Fig. 6. Simulation Model and result of the Under Construction Floor of the Building 


\section{Requirement for System Concept for Construction Production}

Construction production differs from general machine production. Parts and units are supplied from different wide-spread fields, the component factories are also distributed geographically, and the different kind of work, such as conveying, processing and assembling, is combined. Moreover, integrate many enterprise which join the construction project is needed. Therefore, it is difficult that an intensive system treats or utilizes each facility's information in such a production style. The parts and packets unified architecture is effective for management in such kind of project based production. Applying the dynamic management architecture for construction production, the following are required.

- Necessity of collaboration of many companies in deferent fields

- Global optimization to avoid conflicts caused by local optimization

- Management of sheared facilities such as cranes

- Correspond to frequent occurrence of operation plan change

- Correspond to frequent occurrence of schedule change

Figure 7 shows the concept of dynamic management architecture for construction production project. Real-time information of parts/units and sheared facilities are gotten from RFID tags through network, and real-time information of workers also obtained from the manager that grasps each worker's status. By comparing with realtime information to the information on the master plan/schedule, a gap between the master plan/schedule and an actual state is detectable. According to the quantity of gap, re-planning/re-scheduling is performed when the system judges it is needed.

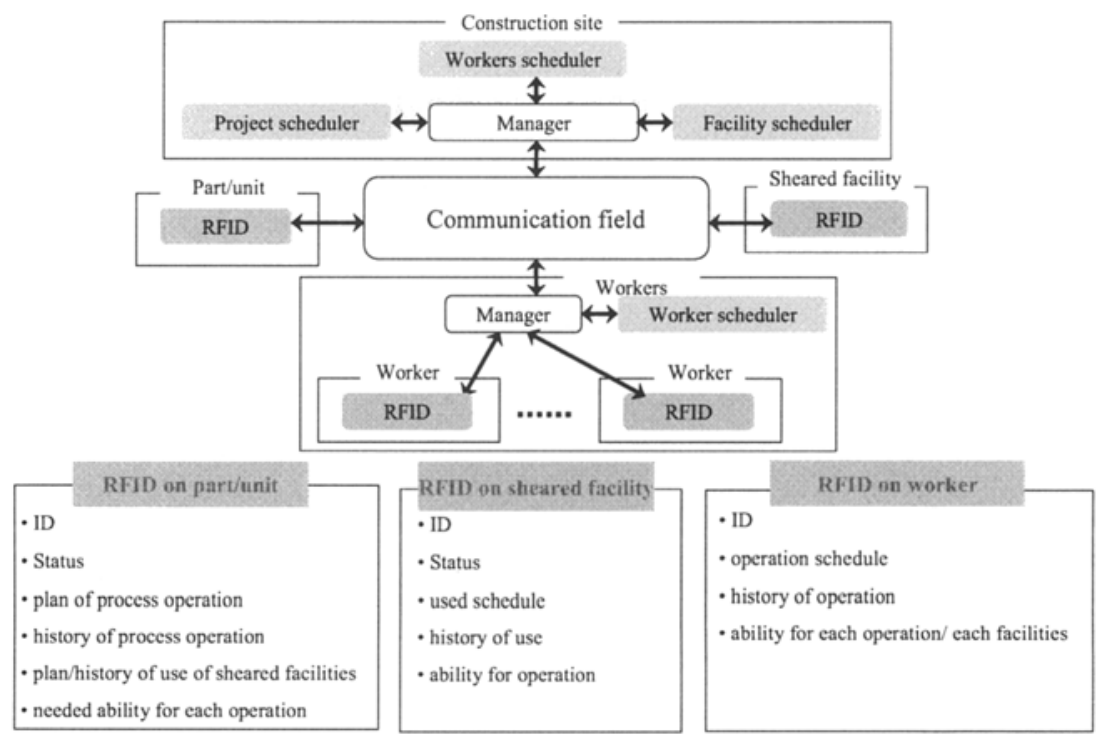

Fig. 7. The concept of dynamic management architecture for construction production 


\section{Conclusion}

The concept and the architecture of the production system with parts and packets unification technology were proposed. In order to treat both scheduling and material handling, not only the information of "what part" and "when exists" but also "where exists" is important. A pilot system is developed and applied to the case studies about the production scheduling and material handling system in a factory and in a construction production site. The results of the case studies show the feasibility of proposed production system using parts and packets unification approach.

\section{References}

1 Ueda, K. (1992). An Approach to Bionic Manufacturing Systems Based on DNA-Type Information, Proceedings of International Conference on Object-Oriented Manufacturing Systems, pp. 305-308.

2 Ranky, P.G. (1992). Intelligent Planning and Dynamic Scheduling of Flexible Manufacturing Cell and Systems, Proceedings of 1992 Japan-U.S.A. Symposium on Flexible Automation, pp. 415-422.

3 Wiendahl, H.P. and Garlichs, R. (1994). Decentral Production Scheduling of Assembly Systems with Genetic Algorithm, Annals of the CIRP, Vol. 43, No. 1, pp. 389-396.

4 Fujii. S., Kaihara. T., and Tanaka, M. (1999). A Distributed Virtual Factory in Agile Manufacturing Environment, Proc. 15th Conference of the International Foundation for Production Research, II, pp.1551-1554.

5 Sugimura, N., Tanimizu, Y., and Yoshioka, T. (1999). A Study on Object Oriented Modeling of Holonic Manufacturing System, Manufacturing System, Vol. 27, No. 3, pp. 253-258.

6 Shirase, K., Wakamatsu, H., Tsumaya, A., and Arai, E. (2005). Dynamic Co-operative Scheduling Based on HLA, Knowledge and Skill Chains in Engineering and Manufacturing - Information Infrastructure in the Era of Global Communications, Springer, pp.285-292.

7 Shiibashi, A. (2002). Introduction and Future Development of Suica Non-contact IC Card Ticketing System. Japan Railway \& Transport Review Vol. 32, pp. 20-27. (http://www.jrtr.net/jrtr32/pdf/f20_shi.pdf).

8 http://www.hitachi.co.jp/Prod/mu-chip/index.html

9 Watanabe, H., Tsumaya, A., Wakamatsu, H., Shirase, K., and Arai, E. (2004). Dynamic Scheduling System with Variable Lot Size Approach Using Parts and Packets Unification, Proceedings of 2004 JAPAN-U.S.A. Symposium on Flexible Automation (JUSFA2004), CD-ROM, JS_038(4 pages).

10 Tsumaya, A., Koike, M., Wakamatsu, H., and Arai, E. (2006). Dynamic Production Management Architecture Considering Preparative Operation, Pre-proceedings of Advanced Production Management Systems Conference 2006 of the IFIP Working Group 5.7, pp.289-294. 